\title{
INTRODUCING EXHALED HUMAN BREATH WATER VAPOR AS ADDITIONAL PROPOSED MECHANISM INFLUENCING SPEED OF SUPERFICIAL CUTANEOUS WOUND HEALING
}

\author{
Abrahám A. Embí BS 1 \(iD) \\ ${ }^{1} 13442$ SW 102 Lane, Miami Florida 33186, USA
}

Received 25 September2021

Accepted 19 October 2021

Published 31 October 2021

\section{CorrespondingAuthor}

Abrahám A. Embí BS, Embi21@att.net

\section{DOI \\ 10.29121/granthaalayah.v9.i10.2021 .4313}

Funding: This research received no specific grant from any funding agency in the public, commercial, or not-for-profit sectors.

Copyright: (C) 2021 The Author(s). This is an open access article distributed under the terms of the Creative Commons Attribution License, which permits unrestricted use, distribution, and reproduction in any medium, provided the original author and source are credited.

\section{ABSTRACT}

The concept that moist wounds heal faster than dry wounds was introduced in 1962. Most recently, in 1990 the concept was revisited with the introduction of a highly permeable wound dressing exposed to water vapors. The latter allows for water as a humidifying agent.

Ideally, acceleration of superficial wound healing had been accomplished by the introduction of a highly water vapor permeable wound dressing. The breathable property allows for water vapor to interact with already present fibrin(ogen) material in blood clots.

This manuscript adds a revised mechanism for the ultimate undisturbed success in cutaneous wound healing, being the dependency on a continuos supply of water vapor. In vitro experiments are introduced showing the cessation of exhaled human breath vapor onto a dry human blood smear as the end point of said interaction. Additionally the experiments were reproduced by exposing the blood smears to steam (water vapor) generated by machinery. In conclusion, exhaled human breath water vapor blown onto a blood clot has the same effect as water vapor emitted by machinery boiling water. Both causing a disappearance of the clot organized fibrin strands into a semisolid gelatinous state. Additionally, discontinuation of the water vapor infusion is also documented triggering a return of organized fibrin strands, albeit of greater intensity.

Keywords: Exhaled Human Breath, Water Vapor, Fibrin Disappearance, Fibrin Appearance, Cutaneous Wound Healing, Wound Healing Acceleration

\section{INTRODUCTION}

The demonstration that a moist environment was beneficial to superficial wound healing was introduced in 1962 (Winter (1962)). Most recent, reports in the literature support a beneficial role in accelerating cutaneous wound repair by using a highly water vapor permeable superficial wound dressings. A stated, the permeable dressing "The breathable film enabled coagulation of the wound exudate, preserving it into a semisolid gelatinous state. This coagulum layer contained an abundant amount of fibrin(ogen) and fibronectin. In wounds occluded with OpSite film, depositions of fibrin(ogen) and fibronectin were less extensive" (Jonkman et al. (1990), Laurens et al. (2006)).

In this manuscript, the continuous presence of water vapor is introduced as an essential factor in maintaining the observed semisolid gelationous state and consequent wound healing acceleration. Human blood smears were prepared as CDC guidleines (Center for Disease Control (CDC) (n.d.)) Video recordings will 
show the essential role of a continuous water vapor presence in perpetuating the process.

\section{MATERIALS AND METHODS}

\subsection{MATERIALS}

Previously prepared dry blood smear on 25x75x1mm glass slide.

Video Microscope Celestron Model \#44348

Water vapor emission from Expresso Coffee IMUSA model GAU-\#18202

Apple McBook Pro Computer with Photo Application.

\subsection{METHODS}

1) Handheld Method Away from Microscope

A previously prepared and dry blood smear slide was held in front of mouth and moist air exhaled sequentially X5. Post breath delivery the, fresh air inhaled though and blown again.

(Figure 1). A: Slide placed in front of open mouth. B: Slide in microscope platform with machine steam source near.

(Figure 2). Recorded images.

\section{Photograph Showing Handheld Approach for Exhaled Breath}

A

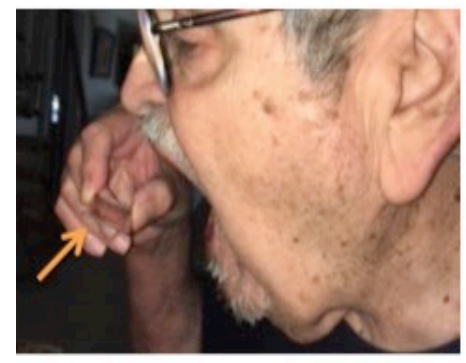

B

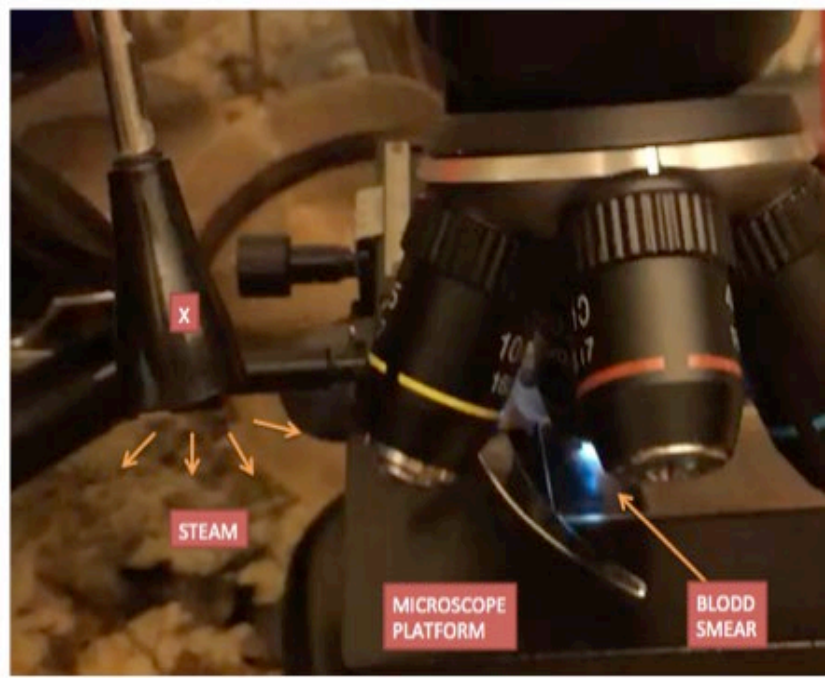

Figure 1 Showing both methods to transfer water vapor onto a blood smear. A: Orange Arrow pointing at blood smear held in front of open mouth as exhaled breath blown. B: Blood smear placed on microscope platform. Red arrows this time showing water vapor source flow. Post breath delivery, fresh air inhaled though mouth and blown again.

Image in B, expanded in Figure 3 below. 


\section{Results from Blowing Exhaled Human Breath Water vapor}

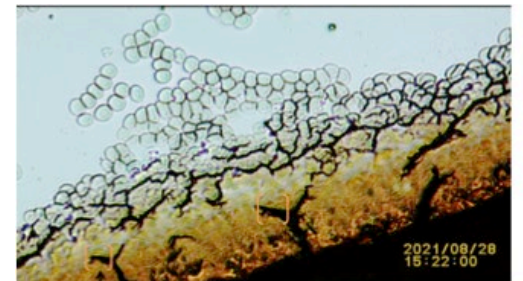

CONTROL

15:22:00

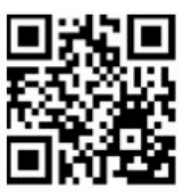

POST BLOWN BREATH X5

FIBRIN DISAPPEAR

$15: 22: 34$

REBOUND

FIBRIN STRANDS

LARGER

$15: 22: 49$

Figure 2 Selected video frames depicting exhaled human breath on fibrin networks. Glass smear on microscope platform showing larger fibrins strands post rebound.

For additional details link to: https://youtu.be/4_2hDup98pQ Or scan QR Code in right upper side of figure.

2) Microscope Platform Near Water Vapor Source

Ancillary Testing Methodology. To rule out Exhaled Breath Ions or particles as cause of fibrinolysis.

The espresso coffee maker was placed in steam mode.

The glass smear placed on the microscope platform.

As soon as water vapor was observed clouding the image, the steam source was moved away.

Images recorded (Figure 3) 


\section{Photograph Showing Video-Microscope Near Water Vapor Source}

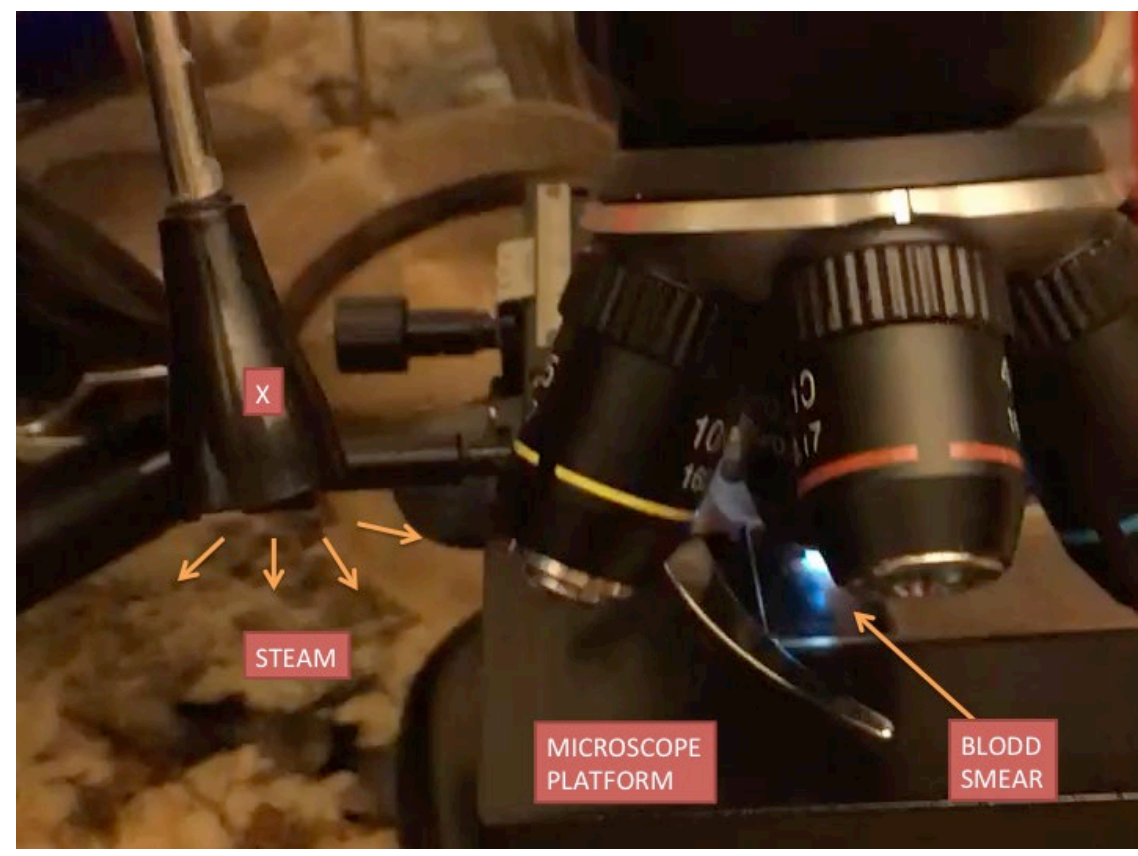

Figure 3 Photographic image of espresso coffee maker next to microscope. Showing X: Hot stem outlet. Orange arrows mimicking steam flow. Notice large orange arrow showing position of blood smear on microscope platform. 3 above.

Images from Machine Emitted Water Vapor on Blood Smear as Shown in Figure
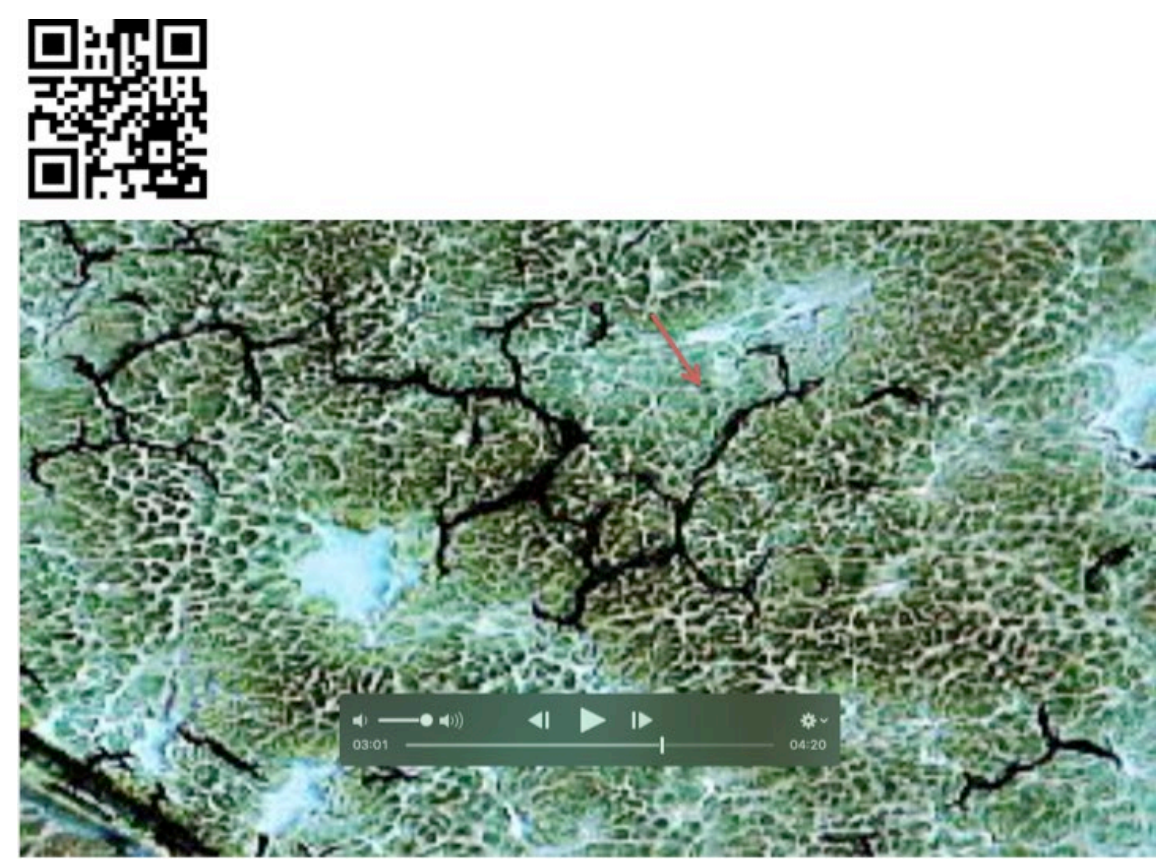

Figure 4 Slide on microscope- Machine steam source near. Red arrow fibrin strands reappearing as water vapor stop. For additional detais, please link to:

https://youtu.be/_PIZTkueHZ8

Or Scan QR Code in upper left of image. 


\section{RESULTS}

The Gelatinous Coagulum Reversal and Fibrin Increase

The images below demonstrates a control image (Figure $5+$ Video) and a post cessation of water vapor infusion (Figure $6+$ video), the noticeable increase in fibrin presence post cessation of water vapor infusions could be seen by comparing red arrows in Figure 5 and Figure 6 below.

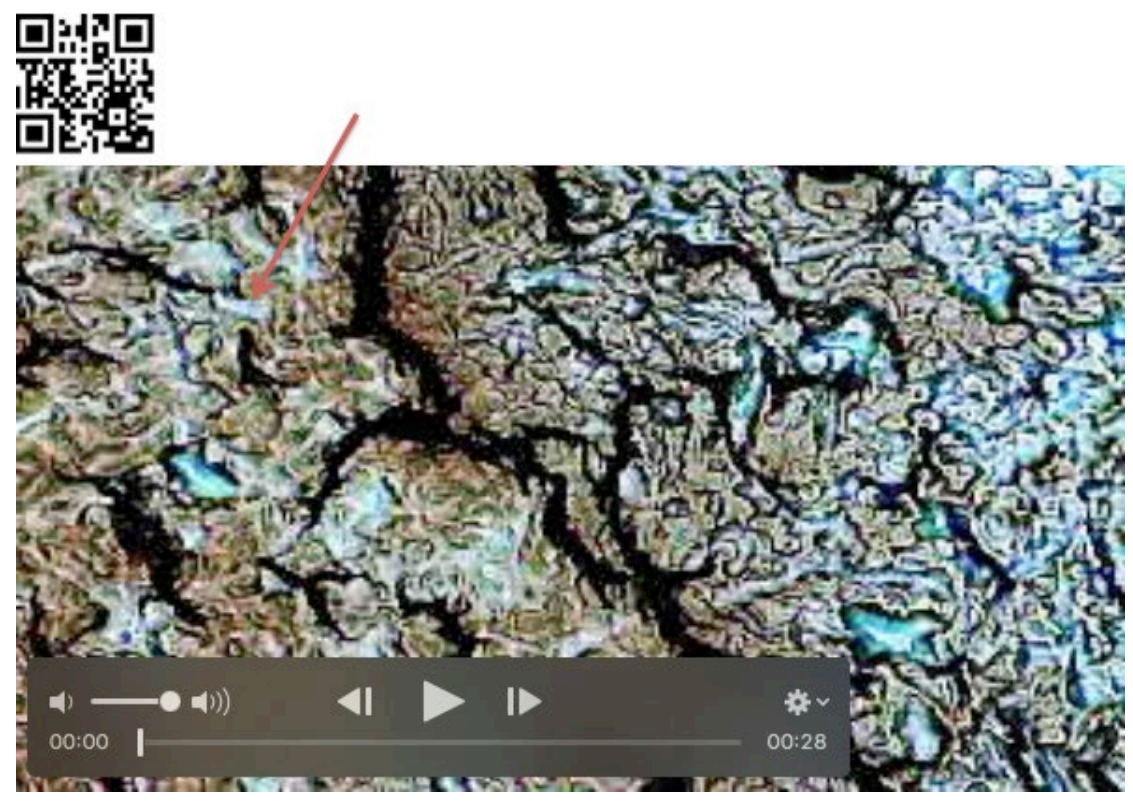

Figure 5 Frame 00:00 Red arrow pointing at initial fibrin network prior to exhaled human breath maneuver. For additional details, please link to: https://youtu.be/QEFiGMYPNuE

Or Scan QR Code in left upper corner.
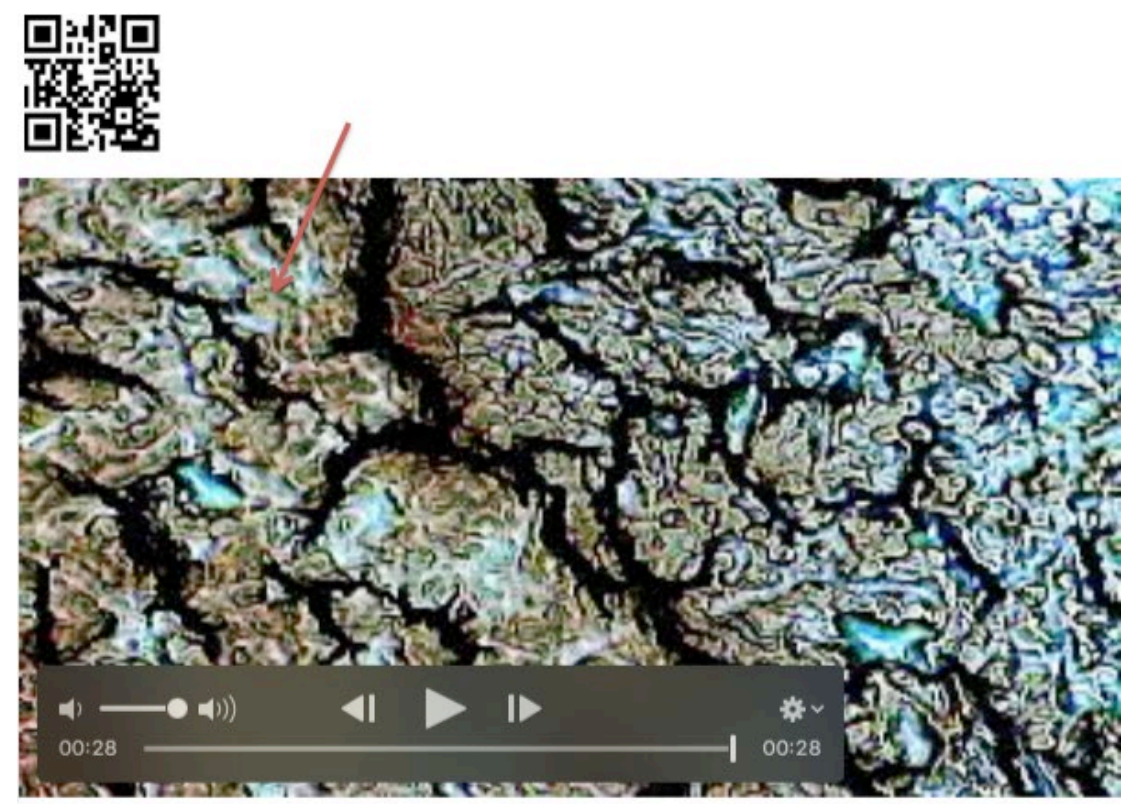

Figure 6 Frame 00:28 Red arrow pointing at increased fibrin network intensity post exhaled human breath maneuver. For additional details, please link to: https://youtu.be/QEFiGIMYPNuE

Or Scan QR Code in left upper corner. 


\section{Demonstration of Reversal of Gelatinous Coagulum into Fibrin Strands During Evaporation}

The image below (Figure $7+$ video) confirms that when the gelatinous coagulum is sandwiched between two slides, the lack of evaporation preserves the coagulum. As soon as the top slide of the sandwiched is removed, there is video evidence of a return of the actual fibrin structures.

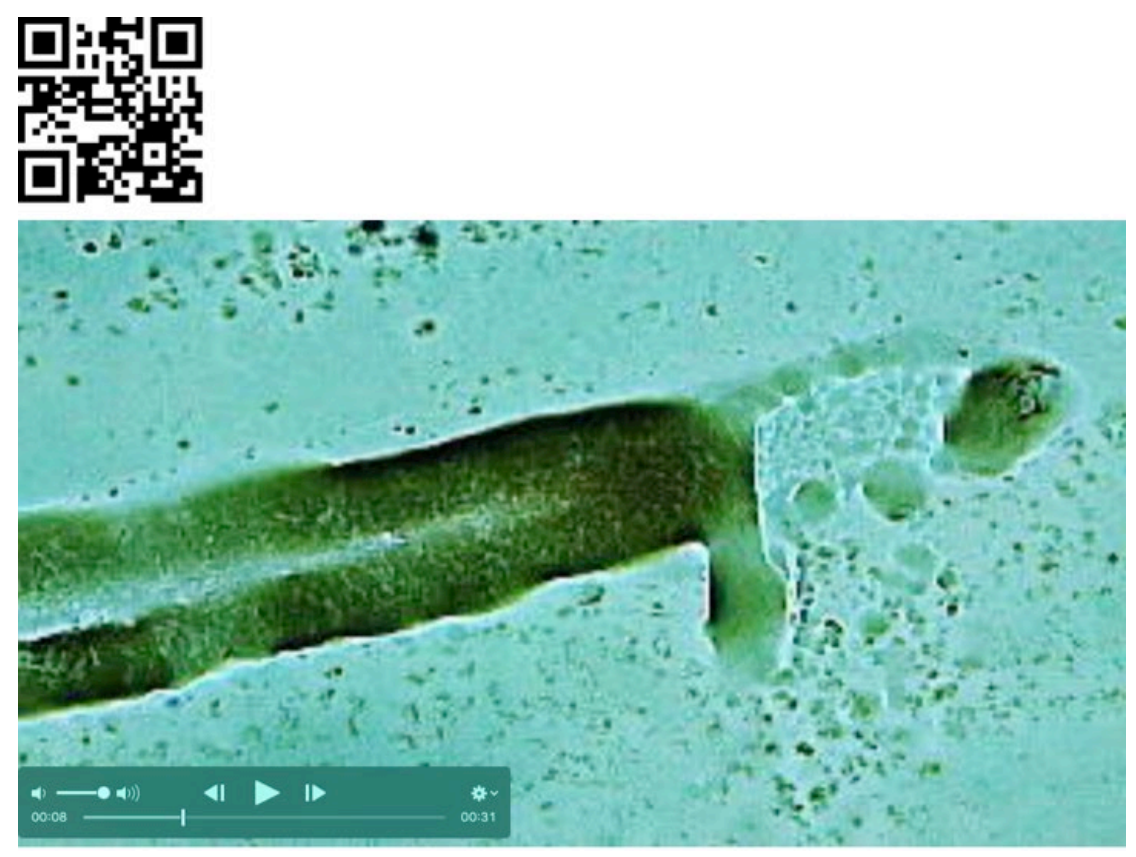

Figure 7 Frame 00:08 Post blown breath - "wet stage"- Gelatinous coagulum was immediately trapped by second glass slide. Showing amorphous mass. Top slide removed and due to evaporation the coagulum reversing back showing fibrin. For additional details, please link to: (See video link below)

https://youtu.be/npopmlrsm3A Or Scan QR Code in left upper corner.

See difference from frame 00:56 at end of video-

\section{DISCUSSION/CONCLUSION}

A mechanism in introduced where a continuous supply of water vapor is essential for the maintenance of a gelatinous coagulum needed for accelerating superficial wound healing. The evidence shows that as soon as there is a cessation of water vapor the coagulum undergoes evaporation and it reverses back to its fibrin network architecture. Previous published statements should be modified to read: "Accelerated epithelization under a highly vapor-permeable wound dressing is associated with increased precipitation of fibrin(ogen) and fibronectin; as long as there is a continuous water vapor supply". "Fibrinogen is converted to fibrin, which forms a cohesive network, and provides a temporary support for wound healing. The structural composition of fibrin and the binding of fibrin to cells and proteins highly determine the wound healing process" (Mosesson et al. (2001), Weisel (2015)).

\section{Saliva and Wound Healing?}

Prior publications attribute saliva many tissue factor in speeding oral would healing, stated "recognition of the many roles salivary proteins play in wound healing makes saliva a promising source for the development of new drugs involved in tissue regeneration" (Brand et al. (2014)). 


\section{Moist Environment and Wound Healing}

The evidence presented in this manuscript identifies a moist "saliva-free" environment in speeding wound healing, a continuos water vapor presence is identified as factor in speeding non-buccal superficial wounds.

\section{Additional Suggestions}

Additional research is highly recommended to rule out unforeseen complications from exhaled human breath vapors, such as new infections in the host that could be airborne transmitted (Zheng et al. (2018)). It has been a very interesting and rewarding finding to be able to observe the water vapor interaction with fibrin(ogen).

\section{REFERENCES}

Brand HS, Ligtenberg AJ, Veerman EC (2014). Saliva and wound healing. Monogr Oral Sci.;24:52-60. doi: 10.1159/000358784. Epub 2014 May 23. PMID: 24862594. Retrieved from https://doi.org/10.1159/000358784

Center for Disease Control (CDC) (n.d.). Preparation of thin blood smears Retrieved from

https://www.cdc.gov/dpdx/resources/pdf/benchaids/malaria/malaria_pr ocedures_benchaid.pdf

Jonkman MF, Hoeksma EA, Nieuwenhuis P. (1990) Accelerated epithelization under a highly vapor-permeable wound dressing is associated with increased precipitation of fibrin(ogen) and fibronectin. J Invest Dermatol. Apr;94(4):477-84. doi: 10.1111/1523-1747.ep12874624. PMID: 2179419. Retrieved from https://doi.org/10.1111/1523-1747.ep12874624

Laurens N, Koolwijk P, de Maat MP (2006). Fibrin structure and wound healing. J Thromb Haemost. May;4(5):932-9. doi: 10.1111/j.15387836.2006.01861.x. PMID: 16689737. Retrieved from https://doi.org/10.1111/j.1538-7836.2006.01861.x

Mosesson MW, Siebenlist KR, Meh DA. (2001) The structure and biological features of fibrinogen and fibrin. Ann NY Acad Sci; 936: 11-30. Retrieved from https://doi.org/10.1111/j.1749-6632.2001.tb03491.x

Weisel JW. (2015) Fibrinogen and fibrin. Adv Protein Chem. 2005;70:247-99. doi: 10.1016/S0065-3233(05)70008-5. PMID: 15837518. Retrieved from https://doi.org/10.1016/S0065-3233(05)70008-5

Winter, GD. (1962) Formation of the Scab and the Rate of Epithelization of Superficial Wounds in the Skin of the Young Domestic Pig. Nature.;193:293294. Retrieved from https://doi.org/10.1038/193293a0

Zheng, Y., Chen, H., Yao, M., \& Li, X. (2018). Bacterial pathogens were detected from human exhaled breath using a novel protocol. Journal of aerosol science, 117, 224-234. Retrieved from https://doi.org/10.1016/j.jaerosci.2017.12.009 